This is a peer-reviewed, accepted author manuscript of the following conference paper: Ramadasan, S., Tao, L., \& Dev, A. K. (2019). Vortexinduced-vibration of jack-ups with cylindrical legs in regular waves. In ASME 2019 38th International Conference on Ocean, Offshore and Arctic Engineering: Volume 1: Offshore Technology; Offshore Geotechnics New York: American Society of Mechanical Engineers (ASME). https://doi.org/10.1115/OMAE2019-95764

OMAE2019-95764

\title{
VORTEX-INDUCED-VIBRATION OF JACK-UPS WITH CYLINDRICAL LEGS IN REGULAR WAVES
}

\author{
Sudheesh Ramadasan ${ }^{1}$ \\ Newcastle University in Singapore \\ (Cybermarine Technologies Pte Ltd) \\ Singapore
}

\author{
Longbin Tao \\ University of Strathclyde \\ Glasgow, United Kingdom
}

\author{
Arun Kr Dev \\ Newcastle University in Singapore \\ Singapore
}

\begin{abstract}
A simple mathematical model is developed based on the single-degree-of-freedom analogy and principle of conservation of energy evaluating various modes of VortexInduced-Vibration (VIV) of a jack-up with cylindrical legs in regular waves. Similar to uniform current, mass ratio, damping ratio and mode factor are found to be the important parameters controlling the cross-flow VIV and radius of gyration also for the yaw VIV. Criteria for the initiation of the mentioned VIV modes are developed for the cases of a single $2 D$ cylinder experiencing planar oscillatory flow, four rigidly coupled $2 D$ cylinders in rectangular configuration experiencing planar oscillatory flow and jack-up experiencing regular waves. The newly developed VIV model is validated by a set of experiments conducted in a wind, wave and current flume. The importance of mass damping parameter is further demonstrated in suppressing VIV in regular waves. The mathematical method will equip engineers to consider the effect of VIV due to regular waves in jack-up designs.
\end{abstract}

Keywords: Jack-up, Vortex-induced-vibration (VIV), VIV criteria, VIV suppression.

\section{INTRODUCTION}

It is well known that alternate shedding of vortices about the cylinders due to flow separation causes Vortex-InducedVibration (VIV), which can lead to structural yield and fatigue failures. Comprehensive reviews on VIV are presented by Sarpkaya and Isaacson [1], Blevins [2], Sarpkaya [3], Williamson and Govardhan [4] and Sumer and Fredsøe [5].

VIV in water is characterised by low mass ratio, added mass and significant fluid damping. Khalak and Williamson [6] presented test results in steady flow and stated that the overall range of excitation as well as the shape of the response curve is determined by the mass ratio while the level of response is characterised by the mass damping parameter. Govardhan and Williamson [7] found that the vibration frequency during lock-in is primarily dependent on mass ratio and vibration frequency can reach remarkably large values when mass ratio is in the order of unity, producing a negative added mass. Vandiver [8] proposed an alternative damping parameter $\left(\mathrm{C}^{*}\right)$ to overcome the limitation of mass damping parameter, which shall be multiplied with the amplitude ratio to calculate the lift coefficient and argued that the alternative might be used to characterise VIV at all reduced velocities in the lock-in range.

FIGURE 1 displays a typical jack-up with cylindrical legs performing soil investigation. Floating multi-cylinder structures like semi-submersibles and TLPs are found to experience Vortex Induced Motion (VIM), a low frequency analogue of VIV. Gonçalves et al. $[9,10]$ conducted experimental studies on VIM with a scaled model of the semi-submersible in steady flow and found that inline, crossflow and yaw motions were experienced. It was further reported that vortex-induced-yaw motion (VIY) occurred when vortex shedding frequency about the columns approached the natural frequency of yaw of the semisubmersible.

The spacing between the cylinders is a significant parameter for multi-cylinder VIV as the cylinders are strongly influenced by wake and proximity interference effects. Zdravkovich [11] conducted extensive wind tunnel experiments with two identical cylinders in tandem, side by side and staggered arrangements and concluded that the coupling between cylinders disappeared when the transverse pitch ratio was above 4 , and the wake

\footnotetext{
${ }^{1}$ Contact author: s.ramadasan@newcastle.ac.uk
} 
interference gradually diminished when the longitudinal pitch ratio was greater than 7 . Wang et al. [12] conducted an experimental study of flow around four circular cylinders in square configuration and reconfirmed that for transverse spacing ratios above 4 , the four-cylinder array can be regarded as two isolated parallel rows of two cylinders in tandem. It was further revealed that for large pitch ratios $(\mathrm{P} / \mathrm{D}>5)$, the vortex shedding from all four cylinders is fully synchronised with constant frequency and definite phase relationships. Assi et al. [13] studied the wake induced vibration (WIV) response of a downstream cylinder for various longitudinal pitch ratios and found that for pitch ratios greater than 8, the WIV was progressively reduced and the response corresponded to that of VIV resonance.

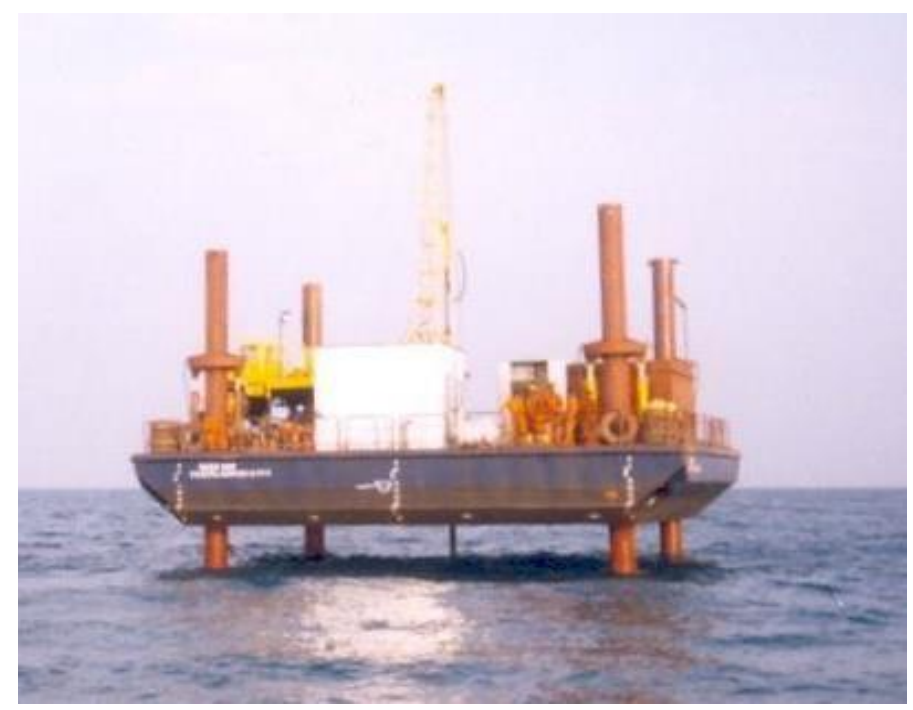

FIGURE 1: JACK-UP WITH CYLINDRICAL LEGS (MS CYBERMARINE TECHNOLOGIES PTE. LTD.)

Jiang [14] numerically studied flow induced transverse vibrations of two tandem cylinders between two parallel walls and found that the cylinders behave as two isolated cylinders for pitch ratios above 8 . Zhao and Cheng [15] performed numerical simulation of VIV of four rigidly coupled square cylinders in a square configuration and reported two modes of vortex shedding; symmetrical, with cylinders on either side shedding vortices symmetrically about the longitudinal centre line, and synchronised, with aggressive lock-in vibrations due to the synchronisation of vortex shedding about all the four cylinders.

Angrilli and Cossalter [16] conducted experiments with a vertical pile in regular waves and found that transverse resonant vibrations at natural frequency are induced by lift forces due to vortex shedding, with lock-in behaviour observed for a wave frequency range of $0.9-1.1 f_{n}$, where $f_{n}$ is the natural frequency. A simplified mathematical model was also proposed for the evaluation of the resonant crossflow VIV in regular waves. Bearman et al. [17] presented a quasi-steady model to predict the lift forces acting on cylinders in waves and oscillating flows. The model was based on a constant Strouhal number of 0.20 and on the assumption that that the lift coefficient is constant over the half cycle of flow. Experiments were conducted with circular cylinders in planar oscillatory flow and the results agreed well with the quasi-steady model at $\mathrm{KC}$ greater than 15 . Sumer and Fredsol [18] carried out experimental studies with an elastically mounter circular cylinder exposed to oscillatory flows covering a vast range of Keulegan Carpenter (KC) numbers, reduced velocities and natural frequencies and concluded that the number of vortices shed per flow cycle for a vibrating cylinder is dependent not only on $\mathrm{KC}$ number, but also on reduced velocity. It was also observed that large crossflow vibrations are possible even at $\mathrm{KC}$ less than 7 caused by the lift forces due to the asymmetry in the strength of the two attached vortices. It was noted that lock-in occurred more than once leading to typical multi peak amplitude response.

McConnell and Park [19] studied the frequency components of lift forces in an oscillatory flow by oscillating a cylinder sinusoidally in still water. A theoretical model was presented which predicted three frequency components for the lift forces in terms of vortex shedding frequency $\left(f_{v}\right)$ and flow frequency $\left(f_{w}\right)$, $f_{v}, f_{v}+2 f_{w}$ and $f_{v}-2 f_{w}$. Velocity ratio, the product of amplitude ratio $(A / D)$ and the natural frequency wave frequency ratio $\left(f_{n} / f_{w}\right)$ was proposed as the significant non-dimensional parameter defining the cylinder VIV and it was demonstrated that the excitation, natural frequency and the added mass were dependent on the velocity ratio. Sarpkaya [20] evaluated the inline and transverse forces acting on a circular cylinder in a harmonically oscillating fluid in a $U$ shaped vertical tunnel and found that transverse forces can be as large as the inline forces. It was observed that the lift coefficient reaches a value as high as 3 at $\mathrm{KC}$ less than 10 , the transverse forces alternate at frequencies that are one to four times the flow frequency and several frequencies are present in a lift cycle.

Verley [21] proposed a simple mathematical model based on instantaneous Strouhal relationship to calculate the lift forces on a cylinder due to vortex shedding in oscillatory flows. The quasisteady model was based on the assumption that the instantaneous velocity is zero at the start of every half cycle and could predict that the lift forces due to vortex shedding spreads over a range of several multiples of wave frequencies on either side of the fundamental frequency. Sarpkaya [22] conducted experiments with smooth and sand roughened cylinders in oscillatory flow for a range of $\mathrm{KC}$ numbers and Reynold numbers $(\mathrm{Re})$ to determine the hydrodynamic forces and first ten harmonics of the lift force acting on the cylinder. It was found that the rms lift coefficient for smooth cylinders reaches a peak value of 1.6, all the ten harmonics are present in the lift force and second and fourth harmonics are predominant in the drag-inertia dominated regimes. Maull and Milliner [23] studied unidirectional sinusoidal flow past a circular cylinder in a $\mathrm{U}$ tube for $\mathrm{KC}$ numbers up to 30 and observed lift coefficients of considerable magnitudes between KC numbers of 10 and 15 due to stable lift generation with minimal intermittency. It was found that the lift force components at double, triple and quadruple the flow frequency are maximum at $\mathrm{KC}$ numbers of 13,18 and 25 respectively. Strong lift frequency components at four and five 
times the flow frequency were also observed at KC 13 and 25 respectively.

Hayashi et al. [24] conducted laboratory investigation to study the dynamic transverse response of a cylinder in planar oscillatory flow and observed twin peaks in the transverse VIV amplitude response similar to that observed for vertical circular cylinder in waves. They have presented a simple mathematical model derived based on the equations of motions and demonstrated the importance of frequency ratios and the inverse proportionality of the amplitude ratio with mass-damping or stability parameter. It was concluded that the VIV response of the cylinder is a function of its ratio of natural frequency to wave frequency, $\mathrm{KC}$ number and damping coefficient in air. Chaplin [25] conducted experimental studies on smooth and rough cylinders at high Re in planar oscillatory flow. The Re were in the range of 20000 to 280,000 and the $\mathrm{KC}$ from 6 to 20 and it was observed that the lift coefficients are in close agreement with that of vertical cylinders in waves. Sumer et al. [26] did span wise correlation measurement for an elastically mounted cylinder exposed to oscillatory flow and undergoing VIV in transverse direction at a $\mathrm{KC}$ of 10 and observed that during lockin, the correlation continuously increased with the amplitude ratio till the maximum value of 0.60 .

The simplest analytical model is the harmonic model [2]. The limitation of the harmonic model is that it cannot capture the lift amplitude interaction or the self-limiting nature of VIV. The simplest nonlinear model is the one which incorporates the amplitude dependence of the lift by means of a three term polynomial fit to experimental data [27]. There are also more complex wake oscillator models which represent the fluid behaviour as a nonlinear self-excited oscillator [28, 29]. The harmonic model was used to develop criteria [2, 30] for the occurrence of VIV in steady flow. Vickery and Watkins [31] derived the dependence of response amplitude on reduced damping by considering the energy balance between excitation and damping at resonance, and presented conditions of similarity between model and prototype for VIV experiments.

Self-elevating platforms or jack-ups with cylindrical legs are usually deployed in shallow and intermediate waters. Cylindrical legs, typically having diameters from $0.50 \mathrm{~m}$ to 4.00 $\mathrm{m}$ can potentially lead to large resonant vibration and lock-in in lateral and yaw directions of the jack-ups when the lift frequency approaches the unit's natural frequency. Such VIV can amplify the mean drag acting on the legs, result in high static and cyclic stresses and eventually lead to yield and fatigue damages. Nicholls-Lee et al. [32] pointed out the instability of the jack-ups in high tidal currents and associated VIV causing large topside motions leading to abortion of operations. Thake [33] described the VIV experienced by jack-ups working in deep and fast currents, and further stated the lack of established design guidelines to address this issue and the need for developing new methods. The potential modes of VIV of the jack-up in regular waves are illustrated in FIGURE 2.

There are limited model tests results on jack-ups available in the published literature and almost none of them pertains to jack-up VIV. Bennett Jr and Patel [34] tested the scaled down model of a jack-up to understand the dynamics in regular waves and confirmed that the behaviour was similar to a classical single-degree-of-freedom (SDOF) system. Johnson and Patel [35] and Grundlehner [36] presented the tests carried out with the free and restrained jack-up model in the elevated condition and evaluated the dynamic amplification.

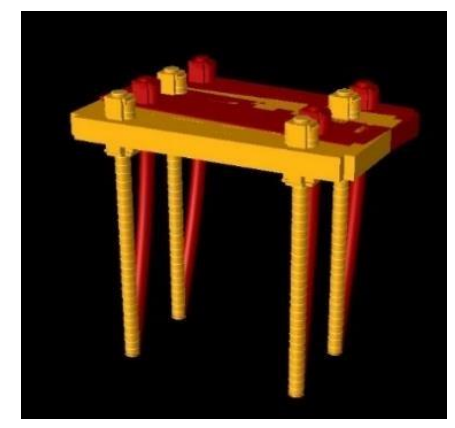

a)

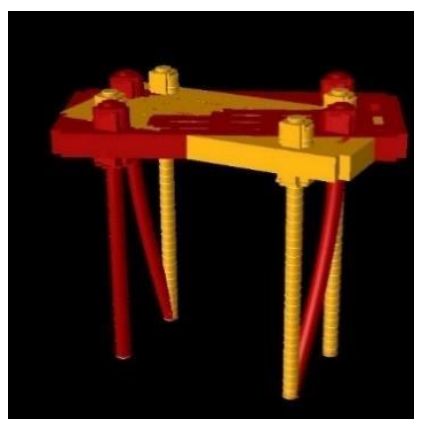

b)
FIGURE 2: JACK-UP VIV MODES IN REGULAR WAVES, a) CROSSFLOW (SWAY), b) YAW (TORSIONAL)

The main objectives of the paper are to understand the VIV of the jack-up with multi-cylindrical legs in regular waves by means of model tests and to develop simple mathematical models to evaluate the various VIV modes. Criteria are proposed for predicting the occurrence of the various VIV modes of jackups in regular waves. The simplified mathematical approach is intended to enable practicing engineers to account for the effect of the VIV in the design stage of jack-ups. The comprehensive experimental results presented will also serve as a benchmark for the future research on the subject.

\subsection{Theoretical Evaluation}

\section{D Circular Cylinders VIV in Planar Oscillatory Flow}

\section{Crossflow / Sway VIV of a Single Cylinder}

The crossflow resonant response amplitude, $y_{O}$ of a lightly damped linear mass spring SDOF cylinder under dynamic excitations can be expressed as [37],

$$
y_{0}=\frac{F L O}{C \omega_{N}}
$$

where $F_{L O}, C$ and $\omega_{N}$ represent the amplitude of the excitation force, damping coefficient and natural angular frequency respectively. The product $C \omega_{N}$ is the dynamic damping stiffness and controls the resonance amplitude.

Since lift frequency is a multiple of wave frequency, the oscillatory lift excitation $\left(F_{L}\right)$ in planar oscillatory flow can be expressed as,

$$
F_{L}={ }_{2}^{1} \rho C D[U \sin (\omega t)]_{0}^{2} L \sin (n \omega t)
$$

where $\rho, C_{L}, D_{1} U_{0}, L, \omega_{1} t$ and $n_{L}$ denote the density of the fluid, oscillatory lift coefficient, diameter of the cylinder, 
amplitude of flow velocity, length of the cylinder, oscillatory flow angular frequency, time instant and lift frequency factor respectively. The above equation satisfies the requirement of oscillatory flow that the lift force should be zero when the flow velocity is zero.

The lift frequency factor $\left(n_{L}\right)$ can be defined as the ratio of the fundamental lift frequency $\left(\omega_{L}\right)$ with the flow frequency,

$$
n_{L}=\frac{\omega \underline{L}}{\omega}
$$

Equation (2) when subjected to further trigonometric operation,

$$
\begin{aligned}
& F={ }^{1} \rho \underline{C} D U^{2} L[2 \sin (n \omega t)-\sin (\langle n \\
& \left.\begin{array}{ccc}
L & 8 & L \\
\sin \left(\left\langle n_{L}+2\right\rangle \omega t\right)
\end{array}\right] \\
& -2\rangle \omega t)-
\end{aligned}
$$

It can be seen from Equation (4) that lift force in oscillatory flow has three frequency components which are higher harmonics of flow frequency; $\left\langle n_{L}-2\right\rangle \omega, n_{L} \omega$ and $\left\langle n_{L}+2\right\rangle \omega$.

Assuming that during resonance the cylinder responds to fundamental lift frequency only,

$$
F_{L}={ }_{4}^{1} \rho \subseteq U_{L}^{2} L \sin (n \omega t)
$$

The damping coefficient can be expressed in terms of a damping ratio $(\zeta)$ as,

$$
\zeta=\frac{C}{2 \sqrt{M K}}
$$

where $M$ and $K$ represent the mass and inline stiffness respectively of the cylinder.

During crossflow resonance or lock-in,

$$
\underset{L}{\omega=} \underset{L}{\omega=} \omega \quad=\sqrt{L}^{K-}
$$

Defining mass ratio $\left(\mathrm{m}^{*}\right)$ of a cylinder as the ratio of mass over displaced mass,

$$
m^{*}=\frac{M}{\rho_{4} D L^{2}}
$$

Defining $\mathrm{KC}$ number $(K C)$ of the flow as,

$$
K C=\frac{U_{0}}{f_{\omega} D}
$$

where $f_{\omega}$ represents the frequency of the flow.

Substituting Equations (5) to (9) in (1) and assuming that the cylinder responds to only fundamental lift frequency during resonance, the amplitude ratio of the crossflow VIV response can be derived as,

$$
\frac{\underline{y} \underline{\underline{O}}}{D}=\frac{\underline{C}_{\underline{L}} \frac{K C^{2}}{8 \pi^{3} n_{L}^{2}} \zeta \mathrm{m}^{*}}{{ }^{*}}
$$

It can be seen from Equation (10) that the response amplitude ratio of a cylinder undergoing crossflow VIV in a planar oscillatory flow is inversely proportional to the product of mass and damping ratios (mass damping parameter) similar to the steady flow. The KC number dependence of the crossflow VIV is also visible from the presence of lift coefficient, lift frequency factor and $\mathrm{KC}$ number in the above expression.

A criterion for the occurrence of the crossflow VIV can be derived by considering $1 \%$ amplitude ratio [30].

$$
\zeta m^{*} \leq \frac{25 C_{L} K C^{2}}{2 \pi^{3} n_{L}^{2}}
$$

Considering the $\mathrm{KC}$ number of 10 at which the maximum lift is observed, corresponding maximum stationary cylinder lift coefficient of $3[20,38]$ and corresponding lift frequency factor of 2 [39], the crossflow VIV criterion in planar oscillatory flow can be,

$$
\zeta m^{*} \leq 30.23
$$

It can be seen from Equation (12) that VIV in planar oscillatory flow can be initiated at a much higher mass damping parameter than steady flow. Hence structures which don't experience crossflow VIV in steady flow may respond aggressively under planar oscillatory flow conditions.

\section{Crossflow VIV of a system of four $2 D$ cylinders in rectangular configuration}

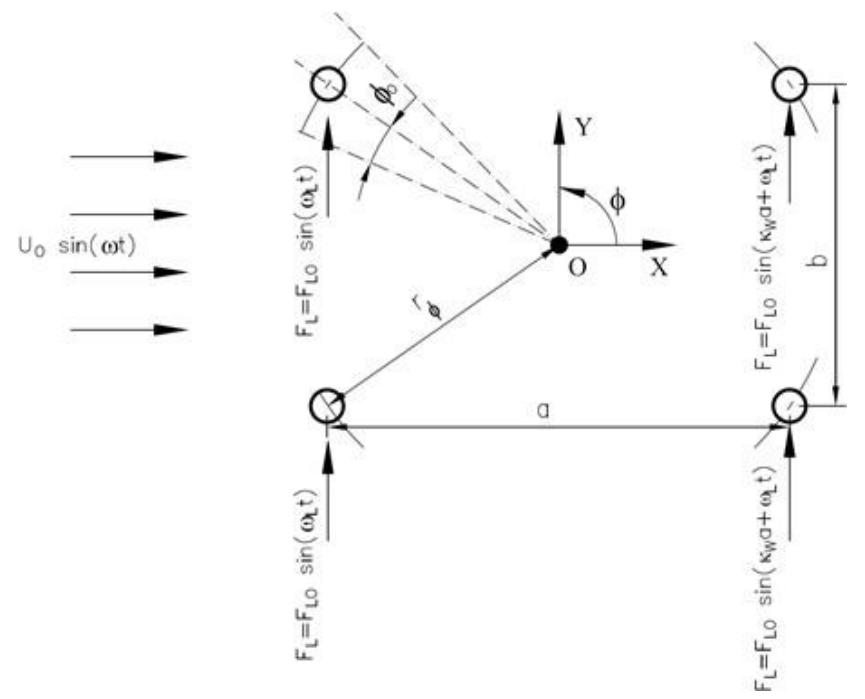

FIGURE 3: SWAY DUE TO CROSSFLOW EXCITATION IN PLANAR OSCILLATORY FLOW

The sway excitation due to lift in a planar oscillatory flow as shown in FIGURE 3 can be expressed as,

$$
\begin{aligned}
& F=2 \times \underset{L}{2} C_{L} D L U U_{0}^{2}\left\{[\sin (\omega t)]^{2} \sin (n \omega t)+[\sin (\omega t+\right. \\
& \left.\left.\left.k_{W} a\right)\right]^{2} \sin \left(n_{L}\left\langle\omega t+k_{W} a\right\rangle\right)\right\}
\end{aligned}
$$


where $a$ is the inline or longitudinal cylinder spacing and $k_{W}$ is the wave number.

Considering fundamental lift frequency only,

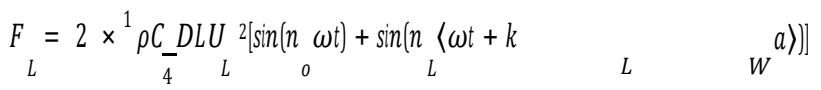

Which can be further simplified as,

$$
F_{L}=2 \times \underset{2}{\rho C_{-}^{1} D L U_{L}} \quad 0^{2 \cos \left(n \quad{ }_{L}{ }^{{ } W^{a}}\right)} \sin \left(\quad \left\langle\omega t+\frac{\left.\left.{ }^{k} W^{a}\right\rangle\right)}{2}\right.\right.
$$

The amplitude $\left(F_{L O}\right)$ of the excitation is,

$$
F_{L O}=2 \times \quad \frac{1}{2} \rho C_{L} D L U_{0} \quad{ }^{2} \cos \left(n_{L} \quad \frac{k w a}{2}\right)
$$

Similar to single cylinder, the amplitude ratio of crossflow VIV resonant response can be derived as,

$$
\frac{y_{\underline{O}}}{D}=\frac{C_{L} K C^{2}}{8 \pi^{3} n_{L}^{2} \zeta m^{*}} \cos \left(n \quad \frac{k_{W} a}{L_{2}}\right)
$$

Equation (17) shows the inverse proportionality of the mass damping parameter, the $\mathrm{KC}$ number dependence and the effect of cylinder longitudinal spacing.

Considering a $1 \%$ amplitude ratio, the criterion for the occurrence of the crossflow VIV can be derived as,

$$
\left.\zeta m^{*} \leq \frac{25 C_{L} K C^{2}}{2 \pi^{3} n_{L}{ }^{2} \cos \left(n_{L}\right.} \quad \frac{k W a}{2}\right)
$$

Considering a KC number of 10, the crossflow VIV criterion in planar oscillatory flow becomes,

$$
\zeta m+30.23 \cos \left(n_{\mathrm{L}} \quad \underline{\underline{k} \underline{w} \underline{a}}\right)
$$

\section{Yaw VIV of a system of four $2 D$ cylinders in rectangular configuration}

The amplitude of yaw VIV response $\left(\emptyset_{O}\right)$ of a system of four 2D cylinders in a planar oscillatory flow under crossflow or lift excitation can be expressed as,

$$
\emptyset_{0}=\frac{M \emptyset O}{C \emptyset \omega N}
$$

where $M_{\varnothing 0}$ and $C_{\varnothing}$ represent the amplitude of yaw excitation moment and yaw damping coefficient respectively.

The yaw excitation due to lift $\left(M_{\varnothing}\right)$, considering the vortex synchronisation between cylinders as shown in FIGURE 4 can be expressed as,

$$
\begin{aligned}
& M=2 \times{ }^{1} \rho C_{-} D L U^{2}\left\{[\sin (\omega t)]^{2} \sin (n \omega t)+[\sin (\omega t+\right.
\end{aligned}
$$

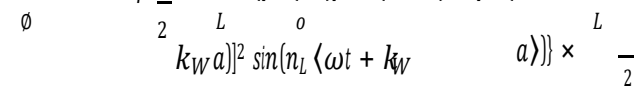
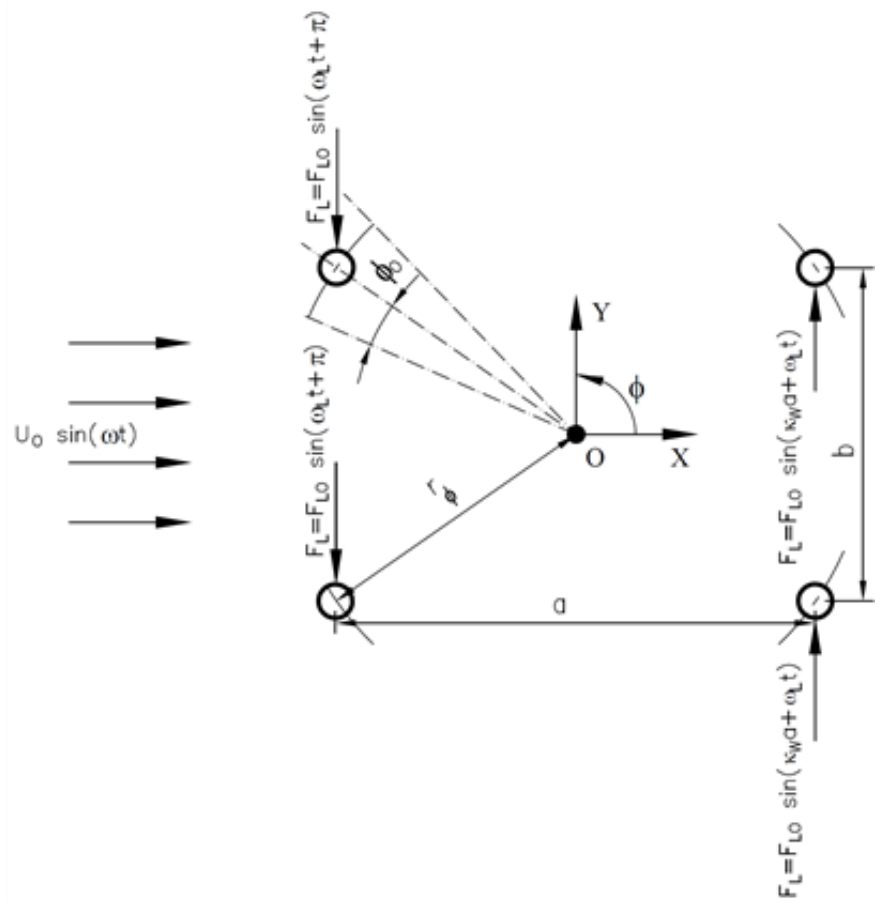

FIGURE 4: YAW DUE TO CROSSFLOW EXCITATION IN PLANAR OSCILLATORY FLOW

Considering the fundamental lift frequency only,

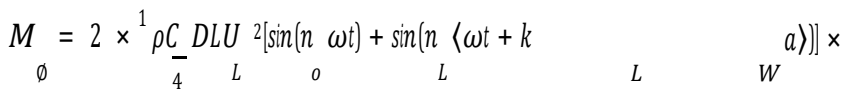

$$
\begin{aligned}
& \frac{a}{2}
\end{aligned}
$$

The amplitude of yaw excitation $\left(M_{\varnothing 0}\right)$ can be derived as,

$$
M_{\emptyset 0} \underset{0}{1} \times_{2} \rho C_{L}^{-D L U_{0}} \quad 2 \cos \left(n_{L} \frac{k_{W} a}{2}\right) \times \frac{a}{2}
$$

During resonance/lock-in under lift excitation,

$$
{ }_{L} \omega={ }_{L} \omega \omega=\omega_{N}=\sqrt{\frac{K \underline{\square}}{I}}
$$

where $K_{\varnothing}$ and $I$ represent the yaw stiffness and yaw moment of inertia respectively.

Considering the $\mathrm{KC}$ relationship, mass ratio, damping ratio and defining the yaw radius of gyration $\left(r_{\varnothing}\right)$ which is also the radial distance of the cylinders from the yaw centre, the amplitude ratio of yaw VIV resonant response can be derived as,

$$
\left.\stackrel{\emptyset}{\underline{0}}=\frac{C_{L} K C^{2} a}{D}=\frac{\underline{k} \underline{w} \underline{a}}{16 \pi^{3} n^{2} \zeta m^{*} \underline{L}^{2} \cos \left(n_{\emptyset}\right.} \quad{ }_{2}\right)
$$

Equation (25) shows the inverse proportionality of the yaw response to the product of mass damping parameter and square of yaw radius of gyration, the $\mathrm{KC}$ number dependence and the effect of leg longitudinal spacing.

Considering a $1 \%$ amplitude ratio, 


$$
\underline{r} \underline{\underline{\emptyset} \underline{\underline{O}}} \underset{100}{\longleftarrow}
$$

The criterion for the occurrence of yaw VIV in planar oscillatory flow can be derived as,

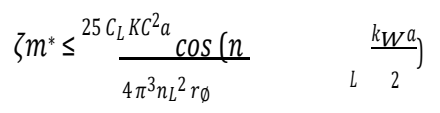

Considering a $\mathrm{KC}$ number of 10 , the yaw VIV criterion in planar oscillatory flow becomes,

$$
\zeta m^{*} r_{\varnothing} \geq 15.12 a \cos \left(n_{L} \quad \frac{k_{W} a}{2}\right)
$$

\section{VIV of Jack-up with four Cylindrical Legs in Regular Waves}

FIGURE 5 illustrates the the typical leg mode shape of the jack-up. The jack-up is idealised as a single degree of freedom system (SDOF) with an elevated concentrated mass in way of the hull leg interface with the legs providing flexural stiffness against lateral deflection.

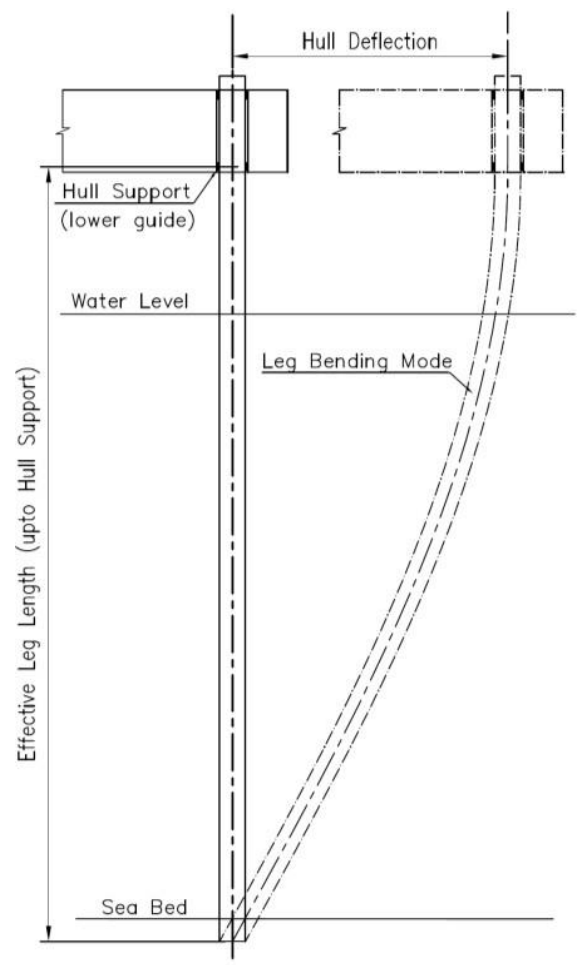

FIGURE 5: JACK-UP LEG MODE SHAPE

The mode shape or deflected profile of the leg of an independent leg type jack-up undergoing resonant vibration can be idealized as a generalised sinusoidal wave. For example, for crossflow vibration,

$$
y_{0}(z)=\left[\frac{Y_{L}}{A \sin (k+B)+E}\right]\left[\underset{L}{A} \sin \left(k_{L} Z+B\right)+E\right]
$$

where $Y_{L}, k_{L}$ represent the crossflow response in way of the hull interface and structural mode number of the leg respectively, $A, B$ and $E$ are constants depending on boundary conditions and $z$ represents the elevation with respect to leg; at leg bottom, $z=$ $O$ and at hull interface, $z=L$.

The effective mass per leg $\left(M_{e L}\right)$ of the equivalent singledegree-of-freedom (SDOF) system of a Jack-up idealised at the hull leg interface level can be expressed based on energy principle [30] as,

$$
M_{e L}=\int_{0}^{L} m z\left\{\frac{A \sin \left(k_{\underline{L}} \underline{\underline{Z}+B)+E^{2}}\right.}{A \sin k_{L} L+B+E}\right] d z
$$

where $m$ represents the mass distribution along the leg.

As evident from the Equation (30), the contributions from the individual mass components, hull, legs, entrapped mass and added mass depend on the leg mode shape and their respective locations along the leg.

\section{Crossflow VIV in Regular Waves}

The effective excitation force $\left(F_{e y}\right)$ of the SDOF system of the jack-up can be expressed based on energy principle [30] as,

$$
\text { ey } \quad F=d \int_{0 y}(z) \frac{A \sin \left(k_{L} \underline{z}+B\right)+E}{A \sin \left(k_{L} L+B\right)+E} d z
$$

where $f_{0 y}$ represents the distribution of the oscillatory lift excitation force along the legs.

The resonant crossflow response $\left(Y_{L}\right)$ of the SDOF can be expressed similar to Equation (1) as,

$$
Y_{L}=\stackrel{\underline{F} \underline{e y}}{C_{e} \omega_{N}}
$$

where $C_{e}$ represents the effective damping coefficient of the SDOF.

The simplest crossflow VIV model for a jack-up can be developed by considering the distribution of oscillatory lift excitation in regular wave, similar to $2 \mathrm{D}$ cylinders as,

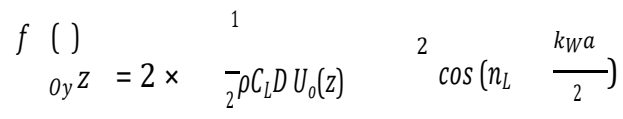

The horizontal velocity distribution along the leg $\left(U_{0}(z)\right)$ in regular waves can be expressed as,

$$
0 \quad U(z)=\frac{\pi H \cosh (k \underline{W} \underline{z})}{T \sinh (k W d)}
$$

where $H_{1} T$ and $d$ represent the wave height, wave period and the effective water depth respectively.

Equations (33) and (34) in (31), $F_{e y}=$
$\quad \rho C_{L} D\left[\frac{\pi H}{T \sinh (k W d)}\right]^{2} \frac{\cos \left(n \frac{k_{W} a}{L}\right)}{A \sin \left(k_{L} L+B\right)+E} \int^{d}[\cosh (k \quad z)]^{2}\left[\begin{array}{l}A \\ \sin (k \quad z+\end{array}\right.$
$B)+E] d z$ 
Equation (32) can be simplified as,

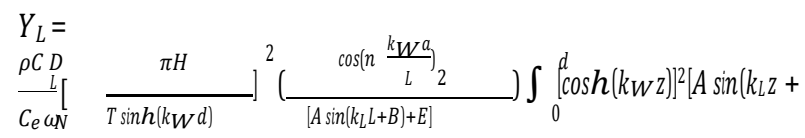

$B)+E] d z$

Defining Keulegan Carpenter number $(K C)$ of the flow at the water level,

$$
K C(d)=\frac{U_{0}(d)}{f_{\omega} D}
$$

Considering the $\mathrm{KC}$ relationship and resonance/lock-in under crossflow excitation,

$$
\begin{aligned}
\frac{Y_{L}}{D}= & \frac{C_{L} K C(d)^{2}}{8 \pi^{3} n_{L}^{2} \zeta m^{*}}\left[\frac{1}{\cosh \left(k_{W} d\right)}\right]^{2}\left(\frac{\cos \left(n_{L} \frac{k_{W} a}{2}\right)}{d\left[A \sin \left(k_{L} L+B\right)+E\right]}\right) \\
& \left.\left.\times \int_{0}^{d_{0} \cosh (k} \quad W^{Z}\right)\right]^{2}\left[A \sin \left(k_{L} Z+B\right)+E\right] d z
\end{aligned}
$$

Equation (38) shows clearly the inverse proportionality with the mass damping parameter, $\mathrm{KC}$ dependence and the effects of longitudinal leg spacing, leg mode shape and horizontal velocity profile on the crossflow VIV. The integration on the right-hand side of the Equation (38) shall be performed only over that extent of leg length experiencing the crossflow excitation, that can be found from the variation of $\mathrm{KC}$ with distance from seabed.

Considering a $1 \%$ amplitude ratio, the criterion for the occurrence of crossflow VIV of a jack-up can be derived as,

$$
\begin{aligned}
& \leq \frac{25 C_{L} K C(d)^{2}}{2 \pi^{3} n_{L}{ }^{2}}\left[\frac{1}{\cosh \left(k_{W} d\right)}\right]^{2}\left(\frac{\cos \left(m_{L} \frac{k_{W} a}{2}\right)}{d\left[A \sin \left(k_{L} L+B\right)+E\right]}\right) \\
& \left.\quad \times \int_{0}^{d} \cosh \left(k_{W} z\right)\right]^{2}\left[A \sin \left(k_{L} Z+B\right)+E\right] d z
\end{aligned}
$$

Considering a KC number of 10, the crossflow VIV criterion of a jack-up in regular wave becomes,

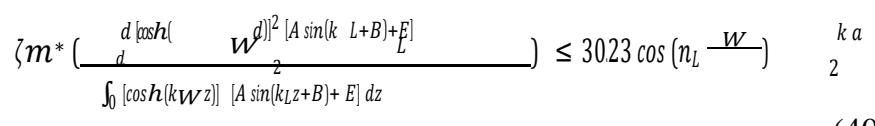

The expression inside parenthesis on the left-hand side can be defined as the mode factor (MF). The product of MF with mass damping parameter can be the effective (or modified) mass damping parameter for a jack-up.

Thus, the criterion becomes,

$$
\zeta m^{*} M F \leq 30.23 \cos \left(n_{L} \quad \frac{k_{W} a}{2}\right)
$$

\section{Yaw VIV in Regular Waves}

Similarly, the total yaw excitation on the jack-up $\left(M_{e \emptyset}\right)$ can be expressed as,

$$
\begin{aligned}
& \begin{array}{l}
M_{e \emptyset}= \\
\rho C D a \\
\frac{L}{2}\left[\frac{L}{T \sinh (k W d)}\right]^{2} \frac{\pi \cos \left(n \frac{k_{W} a}{L}\right)}{A \sin \left(k_{L} L+B\right)+E} \\
B)+E] d z
\end{array} \int_{0}^{d}\left[\cosh \left(k_{W} Z\right)\right]^{2}\left[A \operatorname { s i n } \left(k_{L} Z+\right.\right.
\end{aligned}
$$

The yaw resonant response of the $\operatorname{SDOF}\left(\emptyset_{L}\right)$ can be expressed similar to Equation (20), considering the similarity and vortex synchronisation of the legs.

$$
\emptyset_{L}=\frac{M_{e \emptyset}}{C_{e \emptyset} \omega_{N}}
$$

where $C_{e \emptyset}$ represents the effective yaw damping coefficient of the jack-up SDOF.

Considering KC relationship and lock-in condition, Equation (43) can be further simplified as,

$$
\begin{aligned}
& \frac{\emptyset_{L}}{D} \\
& \left.=\frac{C_{L} K C d^{()^{2}} a}{\left.16 \pi^{3} n_{L}^{2} \zeta m^{*} r^{2} \cos h(k) d x d\right)}\right]\left(\frac{1}{d\left[A \sin \left(k_{L} L+B\right)+E\right]}\right) \\
& \int_{0}^{d}\left[\cosh \left(k \quad w^{z}\right)\right]^{2}\left[A \sin \left(k_{L} Z+B\right)+E\right] d z
\end{aligned}
$$

Equation (44) shows the inverse proportionality of the yaw response with the product of mass damping parameter and the square of the yaw radius of gyration. The KC dependence and the effects of mode shape, velocity profile and longitudinal leg spacing are also captured.

Considering a $1 \%$ amplitude ratio, the criterion for the occurrence of yaw VIV of a Jack-up under regular waves can be derived as,

$$
\begin{aligned}
& \zeta m^{*} r^{2}\left(\frac{d\left[\cosh \left(k_{W} d\right)\right]^{2}\left[A \sin \left(k_{L} L+B\right)+E\right]}{\left.\left.\int_{0}^{d_{L}[\cosh (k} z\right)\right]^{2}[A \sin (k z+B)+E]_{L} d z}\right) \\
& \leq \frac{25 C_{L} K C(d)^{2} a V^{2}+b^{2}}{8 \pi^{3} n_{L}^{2}}
\end{aligned}
$$

where $b$ represents the transverse leg spacing. Considering a $\mathrm{KC}$ number of 10 , the criterion becomes,

$$
\zeta m^{*} M F r^{2}{ }_{\emptyset} \leq 7.56 \cos \left(n_{L} \quad \frac{k \underline{w} \underline{a}}{2}\right) a \sqrt{a} \overline{++2 b \quad 2}
$$

\subsection{Physical Experiments}

\section{Jack-up Model}

A jack-up model with a scale of 1:28 was constructed to suit the tank dimensions. PVC was selected as the model material to satisfy the law of similitude for structural deflections. The leg footings were provided with ball joints to simulate the typical 
pinned boundary conditions. Lead ballast plates were mounted on the model deck to achieve the required elevated load. The elevated mass was free to vibrate in all 6 degrees of freedom (DOF) under the leg excitation. The principal properties of the model are displayed in TABLE 1.

\section{TABLE 1: MODEL PROPERTIES}

\begin{tabular}{|c|c|c|}
\hline Particulars & Prototype & Model \\
\hline Length $(\mathrm{m})$ & 20.00 & 0.71 \\
\hline Breadth $(\mathrm{m})$ & 16.00 & 0.57 \\
\hline Longitudinal leg spacing $(\mathrm{m})$ & 16.00 & 0.57 \\
\hline Transverse Leg Spacing $(\mathrm{m})$ & 12.00 & 0.43 \\
\hline Elevated Load $(\mathrm{kg})$ & 400,000 & 17.94 \\
\hline Leg cantilever length $(\mathrm{m})$ & 27.35 & 0.97 \\
\hline Leg Diameter $(\mathrm{m})$ & 0.94 & 0.034 \\
\hline Leg Material & Mild Steel & PVC \\
\hline Hull Material & Mild Steel & Plastic \\
\hline Water Depth $(\mathrm{m})$ & 20.00 & 0.78 \\
\hline
\end{tabular}

\section{Experimental Setup}

The experiment was conducted in the wind wave current (WWC) tank at the Hydrodynamic Laboratory of the School of Marine Science and Technology, Newcastle University. The WWC tank has a length of $11.00 \mathrm{~m}$, width of $1.80 \mathrm{~m}$ and can accommodate a maximum water depth of $1.00 \mathrm{~m}$, maximum wave height of $0.12 \mathrm{~m}$ and a maximum current of $1.00 \mathrm{~m} / \mathrm{s}$. The blockage ratio is found to be only $3.78 \%$, less than the accepted value of $5 \%$. The model was fixed to the tank bottom, positioned at the measuring section of the WWC tank and was exposed to regular waves at various periods. The model responses were measured by means of two Qualisys motion tracking cameras installed on either side of the model. The experimental setup is illustrated in FIGURE 6.

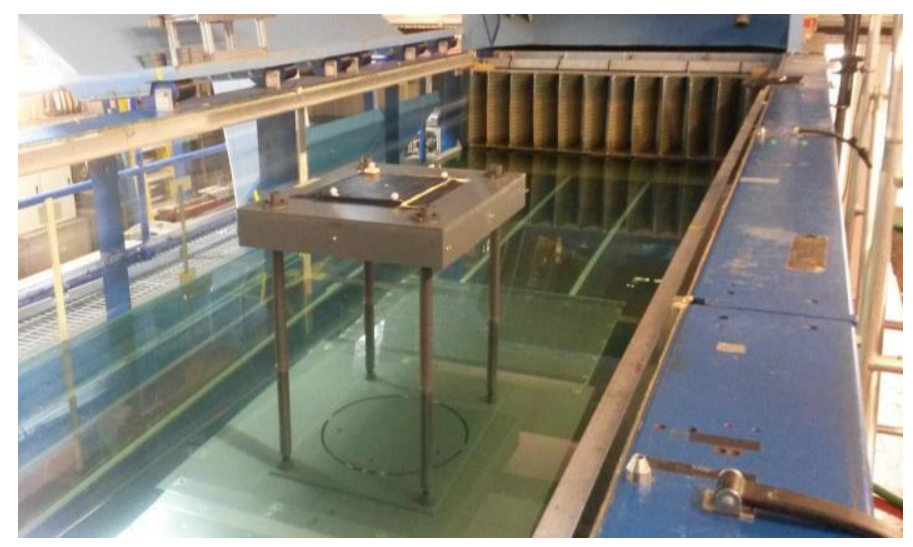

FIGURE 6: EXPERIMENTAL SETUP

\section{Test Procedure}

The tests involved mass tests, stiffness tests, free decay test and response tests. Mass tests yielded the mass, inertia and centre of gravity (COG) details of the model. Stiffness tests verified the stiffness of the model, the effect of ball joint friction on the model stiffness and associated nonlinearities. Natural frequencies, damping properties and the dynamic nonlinearities of the models were established with the free decays tests. Response tests were carried out to evaluate the modes, amplitudes and frequencies of the VIV responses experienced by the model. The response tests were conducted for three elevated loads to cover the operational envelope of a jack-up. However, the response tests for the intermediate elevated load condition (RVL) was carried out for a smaller wave period range corresponding to the crossflow lock-in range only. The details of the test cases are presented in TABLE 2.

TABLE 2: TEST CASES

\begin{tabular}{|c|c|c|}
\hline Test Case & Description & Hull Mass (kg) \\
\hline NVL & No Variable Load & 10.65 \\
\hline RVL & Reduced Variable Load & 14.08 \\
\hline VL & Full Variable Load & 18.05 \\
\hline
\end{tabular}

\subsection{Results and Discussion}

Free decay tests were carried out with the model in air as well as in still water. The tests were intended to establish the natural frequency, added mass, damping and the nonlinearities of the system. As the model was found to exhibit softening nonlinearity at large deflections due to the ball joint friction or support rotational stiffness, the free decay time series was truncated to remove large amplitudes and Fast Fourier Transform (FFT) analysis was carried out to establish the natural frequencies.

TABLE 3 and TABLE 4 summarise the results of sway and yaw free decay tests respectively. From free decay tests, the added mass coefficient of the legs in still water was found to be around 1, approaching to the theoretical value. The corresponding mode shape was found to be that of pinned footing, quarter sinusoid. This showed that for the practical vibration amplitudes, the model legs were vibrating with a mode shape near to that of pinned footing and the effect of the ball joint friction was negligible. It was also revealed that the damping of the system had increased in still water due to the additional fluid damping.

TABLE 3: SWAY FREE DECAY TEST RESULTS

\begin{tabular}{|c|c|c|c|}
\hline \multicolumn{3}{|c|}{ Sway } \\
\hline $\begin{array}{c}\text { Test } \\
\text { Case }\end{array}$ & $\begin{array}{c}\text { Damping } \\
\text { Ratio }\end{array}$ & $\begin{array}{c}\text { Natural } \\
\text { Period (s) }\end{array}$ & $\begin{array}{c}\text { Added Mass } \\
\text { Coefficient }\end{array}$ \\
\hline NVL & 0.040 & 0.82 & 1.35 \\
\hline RVL & 0.044 & 0.93 & 1.29 \\
\hline VL & 0.038 & 1.05 & 1.10 \\
\hline
\end{tabular}

Response tests were carried out by exposing the model to incremental wave periods from the zero heading (0-degree angle of attack). The wave height was chosen to be a constant value of $92 \mathrm{~mm}$. Three loading conditions were considered for the tests to cover the realistic operation scenarios and the practical ranges 
of the mass ratio and the mass damping parameter. The lightest loading condition (NVL) was also tested for various combinations of wave heights and period for a constant $\mathrm{KC}$ number of 5. The responses of the hull along the 6 DOF were measured and recorded as individual time series. The sway and yaw time series were post processed and the root-mean-square (rms) amplitudes and vibration frequencies were calculated for various current speeds. The results of the response tests are summarised in TABLE 5. Added mass is not considered in the mass ratio of the sway mode as the same is found to be negative during crossflow lock-in [7].

TABLE 4: YAW FREE DECAY TEST RESULTS

\begin{tabular}{|c|c|c|c|}
\hline \multicolumn{4}{|c|}{ Yaw } \\
\hline $\begin{array}{c}\text { Test } \\
\text { Case }\end{array}$ & $\begin{array}{c}\text { Damping } \\
\text { Ratio }\end{array}$ & $\begin{array}{c}\text { Natural } \\
\text { Period (s) }\end{array}$ & $\begin{array}{c}\text { Added Mass } \\
\text { Coefficient }\end{array}$ \\
\hline NVL & 0.038 & 0.55 & 0.88 \\
\hline RVL & 0.043 & 0.58 & 0.95 \\
\hline VL & 0.043 & 0.58 & 0.82 \\
\hline
\end{tabular}

TABLE 5: RESPONSE TEST RESULTS, CROSSFLOW/SWAY b) YAW

a)

\begin{tabular}{|l|l|l|l|l|}
\hline \multicolumn{5}{|c|}{ Sway } \\
\hline Case & $\mathbf{m}^{*}$ & MF & yo, rms/D & yo, max/D \\
\hline VL & 6.99 & 2.457 & 0.05 & 0.08 \\
\hline RVL & 5.57 & 2.701 & 0.06 & 0.11 \\
\hline NVL & 4.35 & 3.091 & 0.13 & 0.17 \\
\hline
\end{tabular}

b)

\begin{tabular}{|c|c|c|c|c|c|}
\hline \multicolumn{6}{|c|}{ Yaw } \\
\hline Case & $\mathbf{m}^{*}$ & MF & $\begin{array}{c}r_{\phi} \\
(\mathbf{m})\end{array}$ & $R_{\phi} \phi_{o}, r_{s} / D$ & $\mathbf{R}_{\phi} \phi_{0, \max } / \mathbf{D}$ \\
\hline VL & 7.38 & 5.026 & 0.233 & 0.032 & 0.058 \\
\hline NVL & 4.73 & 3.091 & 0.275 & 0.042 & 0.072 \\
\hline
\end{tabular}

Note: $R_{\phi}$ represents radial distance of leg from yaw centre.

FIGURE 7 shows the variation of the normalised crossflow rms amplitude response plotted with respect to the corresponding reduced velocities. It is evidently shown that the model is highly vulnerable to crossflow VIV with large amplitude lock-in vibrations. The response amplitudes (rms) were found to be around $0.13 \mathrm{D}$ and the maximum response amplitude was observed to be as high as $0.17 \mathrm{D}$.

The peak crossflow response was found to be happening at a reduced velocity of around 5. However, the lock-in regime is found to be very narrow around the peak response. The response amplitude and nominally the lock-in range are found to increase with reduction in elevated load, with the lightest test case exhibiting the largest crossflow response amplitude and lock-in regime. FIGURE 7 also shows the crossflow response of the lightest loading condition in uniform current for comparison. It can be observed that the amplitude response is considerably higher than in uniform current while the lock-in range is considerably smaller. The crossflow response in regular wave is also characterised by the classical twin peaks in the response curve.

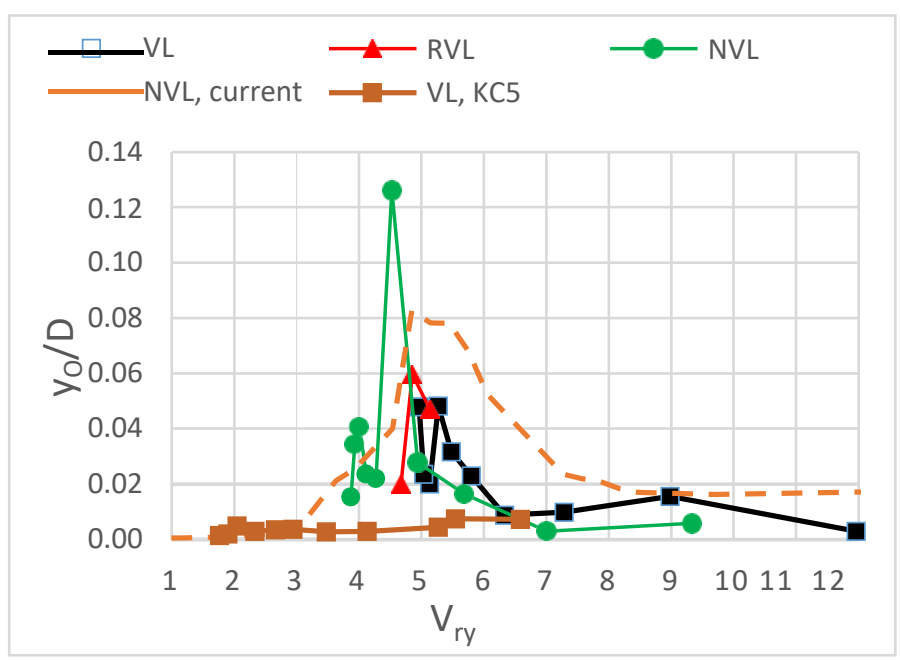

FIGURE 7: CROSSFLOW AMPLITUDE RESPONSE WITH REDUCED VELOCITY $\left(U_{O} / f_{N y} D\right)$

FIGURE 8 shows the variation of the crossflow rms amplitude responses, plotted against the $\mathrm{KC}$ numbers of the flow. It can be observed that the peak crossflow responses correspond to the $\mathrm{KC}$ numbers around 10 . As the $\mathrm{KC}$ number at resonance is roughly twice of the corresponding reduced velocity, it can be inferred that the crossflow response is occurring at an excitation frequency that is double the wave frequency, which corresponds to the fundamental lift frequency at these $\mathrm{KC}$ numbers. It can be observed that the highest response peak corresponds to lock-in with the lift component at double wave frequency while the second peak corresponds to lock-in with the lift component at triple wave frequency. This phenomenon is visible for two test cases, VL and NVL in the figure, while the range of the test case $\mathrm{RVL}$ is too small to capture the second peak.

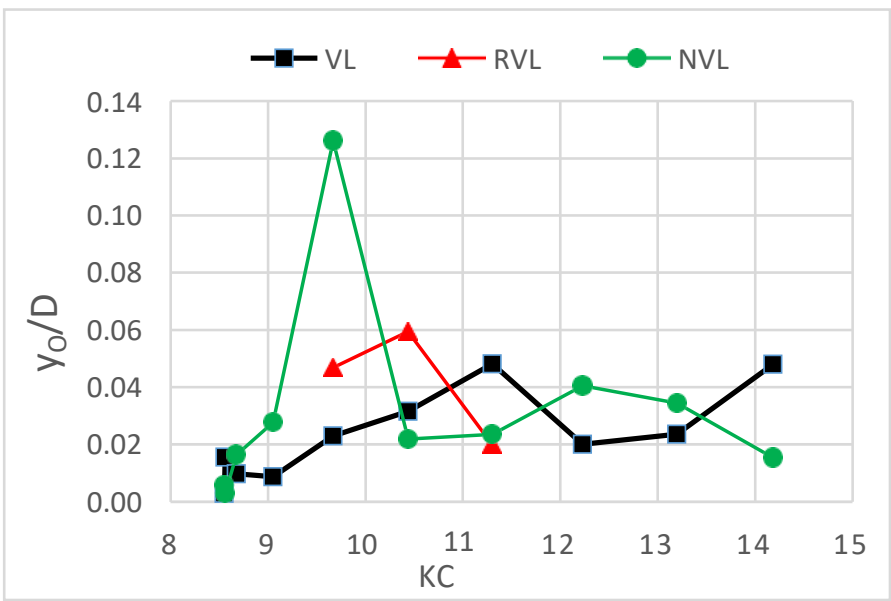

FIGURE 8: CROSSFLOW AMPLITUDE RESPONSE WITH KC NUMBER 
The increase in response amplitude with reduction in model mass validated the inverse proportionality established in the mathematical model. It was observed that the increase in mass ratio not only reduces the amplitude response but also the lockin range. The normalised crossflow rms response amplitude ratios fitted reasonably well in a straight line, when plotted against the inverse of mass damping parameter multiplied with the mode factor (effective mass damping parameter) as shown in FIGURE 9. This demonstrated the significance of effective mass damping parameter in controlling the cross-flow VIV response in regular waves. Thus, the effective mass damping parameter can be considered as a universal parameter for the comparison of crossflow VIV across various mode shapes and water depths in regular waves.

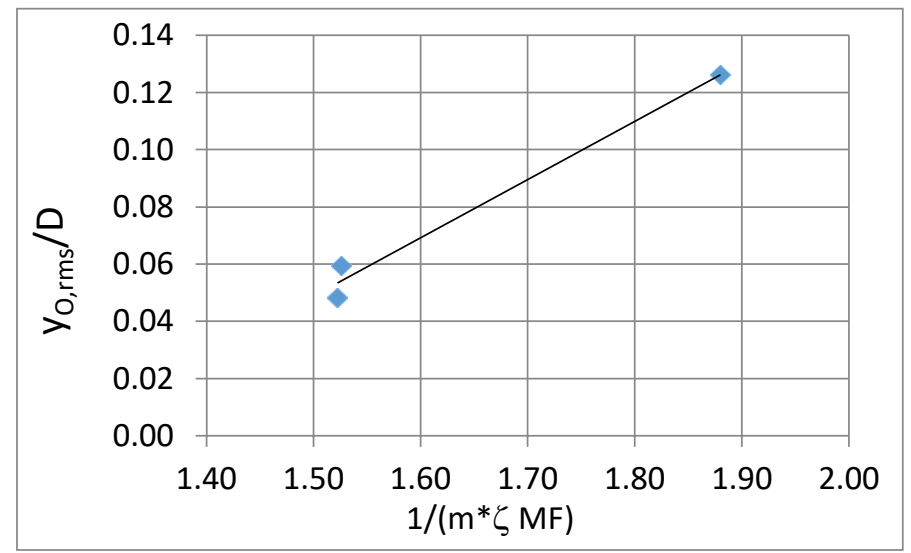

FIGURE 9: CROSSFLOW AMPLITUDE RESPONSE WITH INVERSE OF MODIFIED MASS DAMPING PARAMETER

FIGURE 10 illustrates the crossflow frequency responses of the model normalised with the wave frequency and plotted against the respective $\mathrm{KC}$ numbers for the test cases. It can be observed from FIGURE 10 that the lift excitation is present at multiples of wave frequency as found by many researchers. Further, it is evident during crossflow resonance, the lift frequency is at twice the wave frequency. From the frequency response, it can be observed that the lift excitation is also present at the wave frequency for small KC numbers. The fundamental lift frequency is found to be at $3^{\text {rd }}$ and $4^{\text {th }}$ multiples of wave frequency also at high $\mathrm{KC}$.

FIGURE 11 illustrates the cross-flow frequency responses of the model normalised with the natural frequency and plotted against the corresponding reduced velocities. It can be observed that the natural frequency ratio tends to oscillate around a value of 1 , causing the jack-up to respond to the frequency component of the lift force that is close to its natural frequency. On comparing FIGURE 7 with FIGURE 10, it can be understood that the peak amplitude responses occur at those reduced velocities when the natural frequency ratio is coinciding with or almost near to a value of unity.

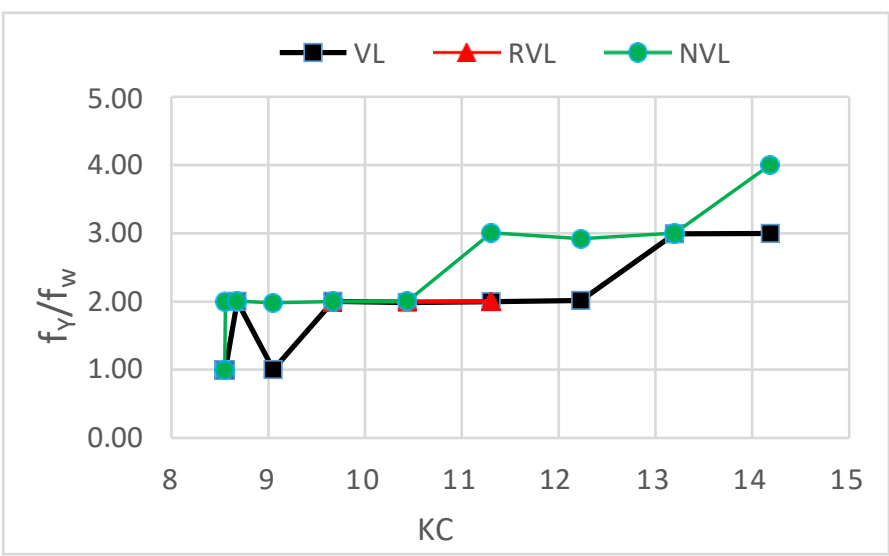

FIGURE 10: CROSSFLOW FREQUENCY RESPONSE NORMALISED WITH WAVE FREQUENCY AGAINST KC NUMBER

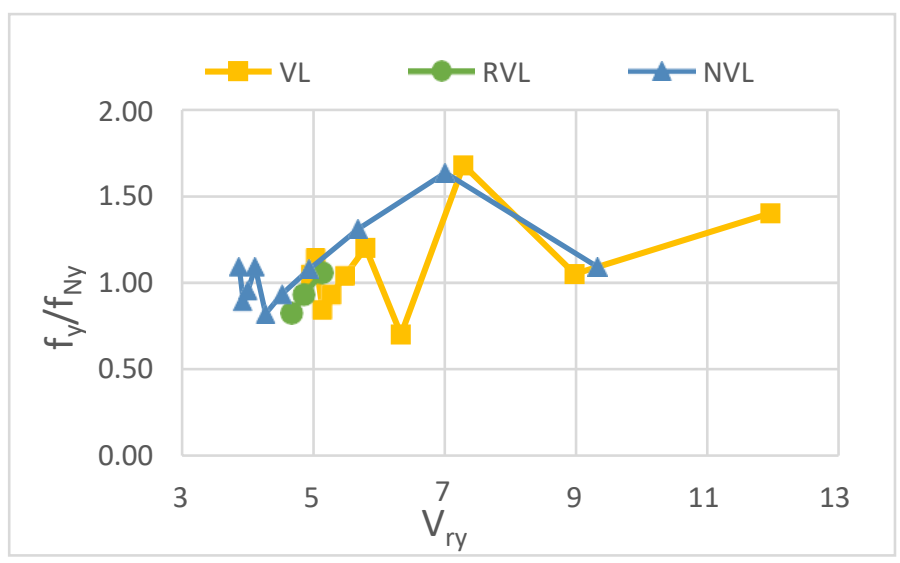

FIGURE 11: CROSSFLOW FREQUENCY RESPONSE NORMALISED WITH NATURAL FREQUENCY AGAINST REDUCED VELOCITY $\left(U_{O} / f_{N y} D\right)$

Experimental observation revealed that the model jack-up experienced torsional or yaw VIV responses also. The yaw amplitude responses as a function of yaw reduced velocity are plotted in FIGURE 12. It can be seen that the peak yaw response

occurs around a yaw reduced velocity less than 5, which demonstrates that the yaw VIV is due to higher multiples of lift excitation. The rms response amplitudes are found to be around $0.04 \mathrm{D}$, with the maximum response amplitude around 0.07D. The lock-in regime is found to be considerably small and triple response peaks are observed for yaw VIV; the range of the test

case RVL was too small to capture the triple peak response. It can be observed that the yaw response is less aggressive than crossflow response in regular waves. The yaw response in regular wave is also found to be less than that in steady current.

FIGURE 13 shows the yaw amplitude response plotted as a function of the $\mathrm{KC}$ number of flow. The response amplitude is found to increase with the reduction in elevated load though there

is no change in the lock-in range. It can be inferred that the increase in mass ratio reduces the yaw amplitude response. The response amplitude ratio of the two load cases, NVL and VL is plotted against the inverse of inertia damping parameter as 
illustrated in FIGURE 14. Inertia damping parameter is the product of mass damping parameter, mode factor and square of yaw radius of gyration. It can be observed that the yaw response increases with the inverse of inertia parameter and it can be considered as the universal parameter determining the yaw VIV response.

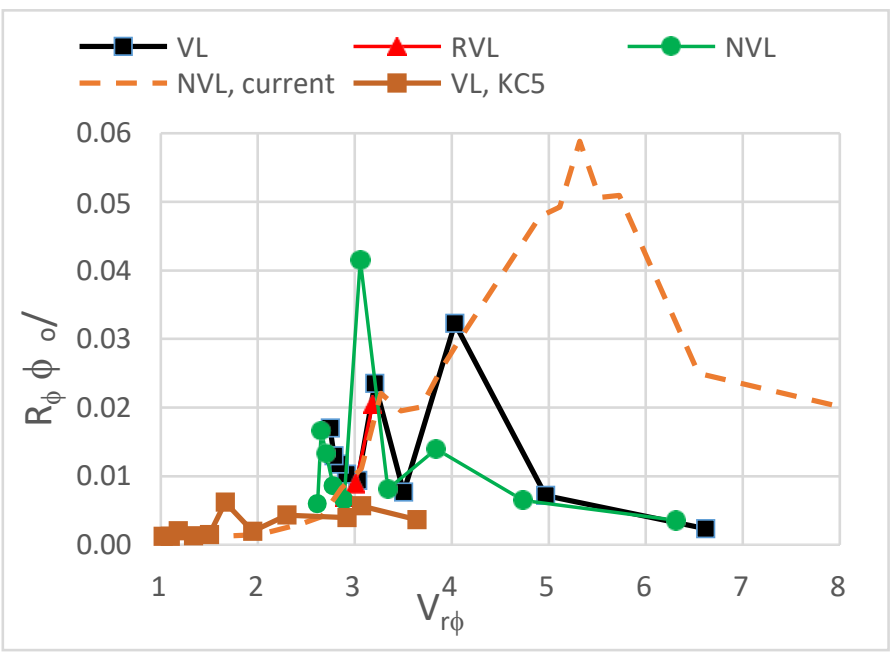

FIGURE 12: YAW AMPLITUDE RESPONSE WITH REDUCED $\operatorname{VELOCITY}\left(U_{O} / f_{N} D\right)$

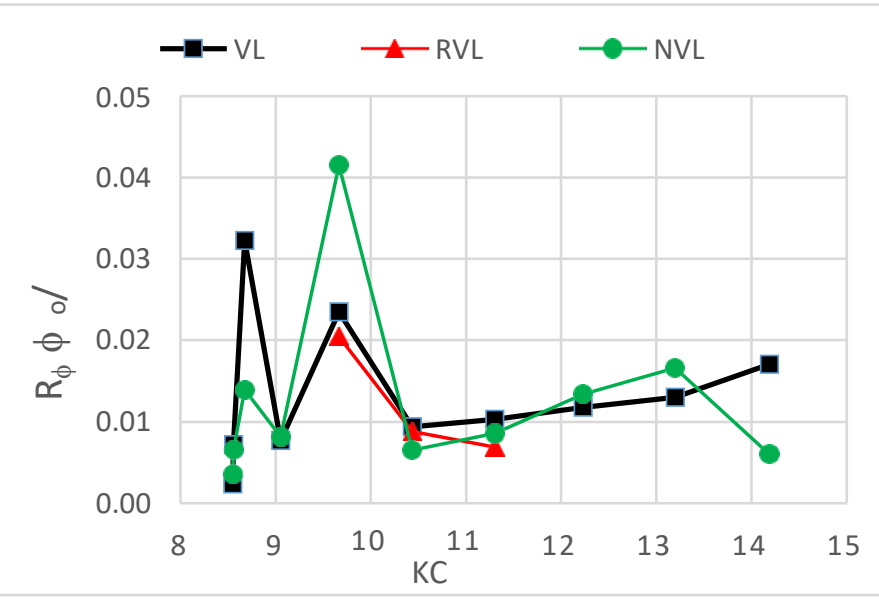

\section{FIGURE 13: YAW AMPLITUDE RESPONSE WITH CORRESPONDING KC NUMBERS}

FIGURE 15 illustrates the yaw frequency responses of the model normalised with the wave frequencies and plotted against the $\mathrm{KC}$ number of flow. It can be observed that the lift excitation is present at multiples of wave frequency, even up to the $5^{\text {th }}$ multiple. It can be inferred from the figure that during the highest yaw resonance peak, the lift frequency is at twice the wave frequency. It can be observed that the lift excitation is present at the wave frequency also for small KC numbers. The lift frequencies are also found to be present even at the $5^{\text {th }}$ multiples of wave frequency at high $\mathrm{KC}$.

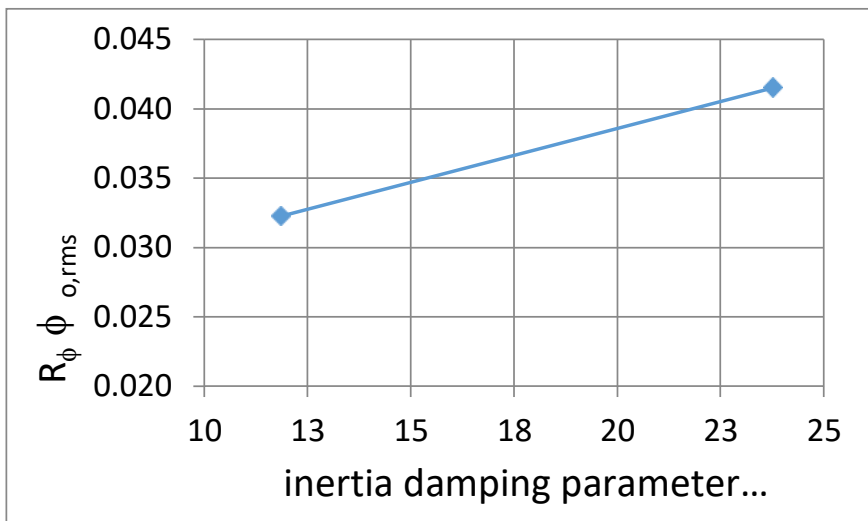

FIGURE 14: YAW AMPLITUDE RESPONSE WITH INVERSE OF INERTIA DAMPING PARAMETER

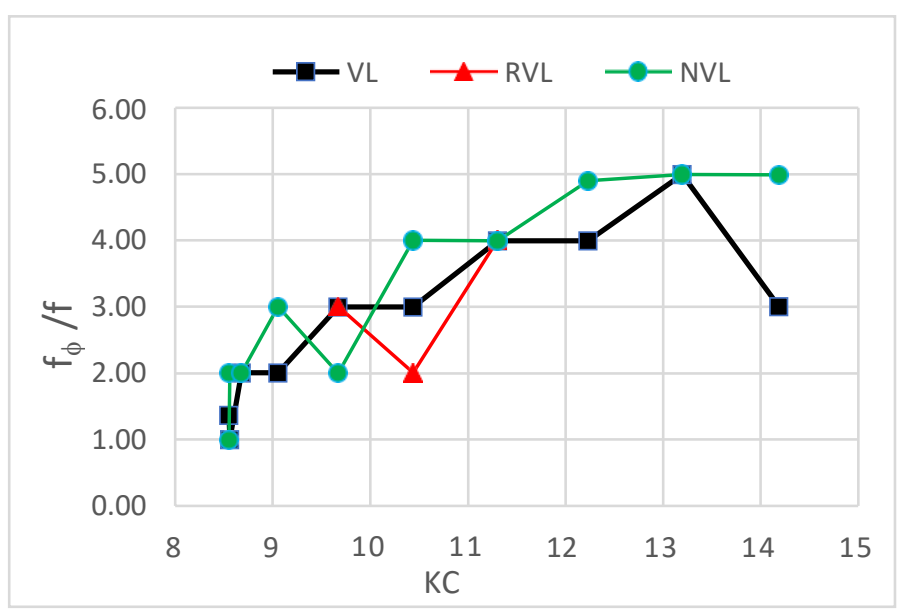

FIGURE 15: YAW FREQUENCY RESPONSE NORMALISED WITH WAVE FREQUENCY AGAINST KC NUMBER

The yaw frequency responses of the model normalised with the corresponding natural frequencies and plotted against the yaw reduced velocities is displayed in FIGURE 16. Similar to crossflow response, the natural frequency ratio is found to oscillate around a value of unity causing the model to respond to that lift frequency component near to its natural frequency. Comparison with FIGURE 12 reveals that the peak amplitude responses occur at those reduced velocities when the natural frequency ratio is near to a value of unity.

The combined crossflow amplitude response of the leg derived from the corresponding crossflow and yaw responses is plotted in FIGURE 17. It is found that the range of the yaw response overlaps with that of the crossflow response particularly for low mass ratios, causing a combined lock-in range. The combined vibrations experience higher response amplitudes than the individual values, especially for low mass ratios. The combined response rms amplitude is observed to be as high as $0.16 \mathrm{D}$. The mean or steady inline, crossflow and yaw responses were also measured and the same were found to be insignificant as expected throughout the wave periods. 


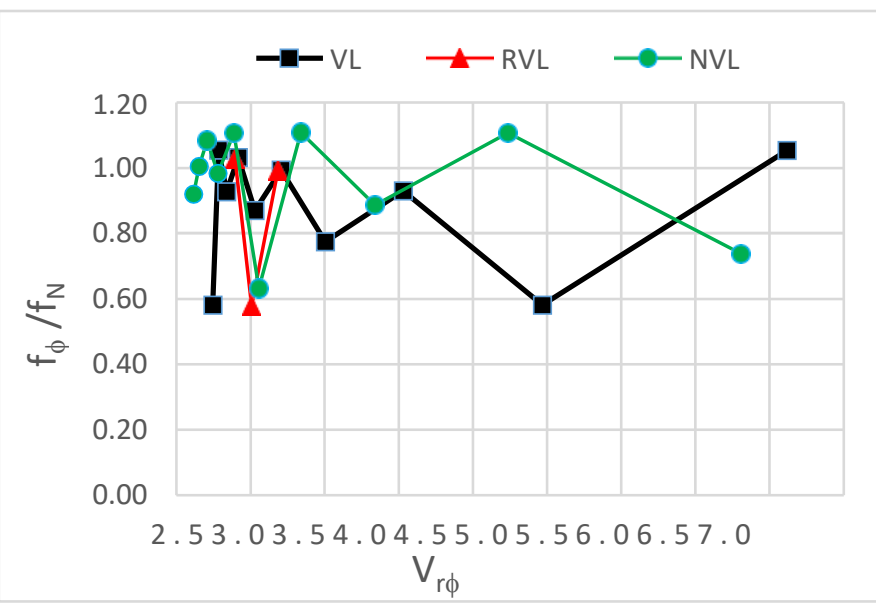

FIGURE 16: YAW FREQUENCY RESPONSE NORMALISED WITH NATURAL FREQUENCY AGAINST REDUCED VELOCITY $\left(U_{O} / f_{N} D\right)$

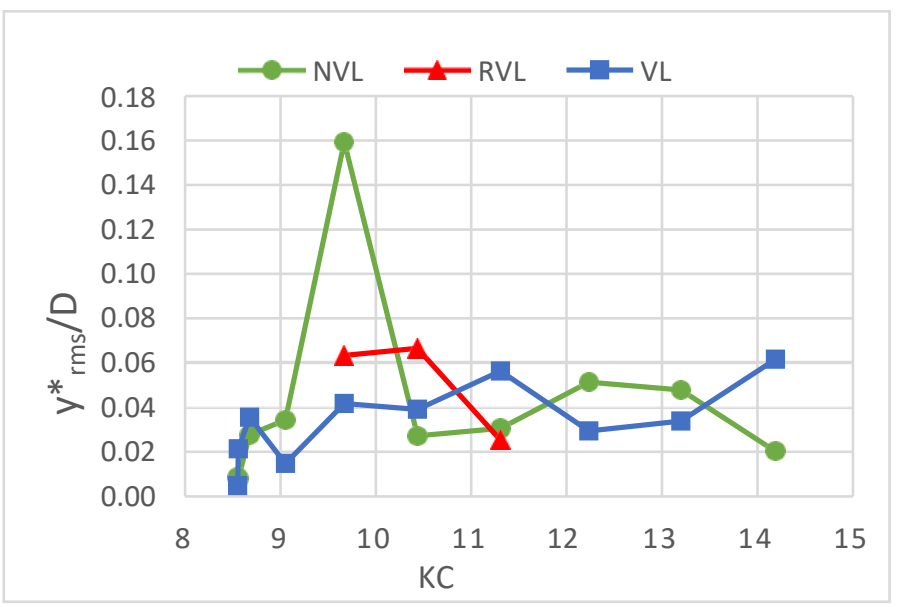

FIGURE 17: LEG CROSSFLOW COMBINED AMPLITUDE RESPONSE DUE TO SWAY AND YAW WITH KC NUMBER

\subsection{Conclusions}

A simple mathematical model is proposed based on the single-degree-of-freedom analogy and the principle of conservation of energy, to evaluate various modes of VIV of a jack-up with cylindrical legs in regular waves. Mass ratio, damping ratio and mode factor are found to be the important parameters controlling the cross-flow VIV of jack-ups. Radius of gyration is found to be the additional vital parameter influencing yaw VIV. Criteria for the occurrence of crossflow and yaw VIV are proposed for the cases of a single $2 \mathrm{D}$ cylinder, four $2 \mathrm{D}$ cylinders in rectangular configuration and a complete jack-up with four cylindrical legs in regular waves.

The model tests demonstrated that the jack-up experienced both crossflow and yaw lock-in vibrations in regular waves with maximum amplitude ratios in excess of 0.1D. The crossflow and yaw VIV are found to occur due to the lift excitation when the lift frequency approaches the sway and yaw natural frequency respectively of the jack-up. The lift force is found to have frequency components at all multiples of the wave frequency, even up to the 5th multiple. Outside the lock-in range, both the sway and yaw modes are found to be excited by lift forces at frequency components other than the fundamental lift frequency, depending on the proximity to the natural frequency. It is also observed that the crossflow response in regular waves is higher than that in uniform current, while the yaw response is lesser.

From the test results, it can be inferred that the jack-ups are highly vulnerable to crossflow and yaw VIV in regular waves, particularly in light operating conditions. The crossflow and yaw responses were also found to reduce with increase in effective mass damping and inertia damping parameters respectively, validating the inverse proportionality in the VIV model. The lock-in range of the crossflow VIV was found to overlap with the lock-in range of yaw VIV, particularly in light operating conditions, causing very high combined leg amplitude response. Based on the test results, it is recommended to maximise the mass ratio of the jack-ups during design and operation.

As the combined response is of very high amplitudes, significant dynamic stresses can be induced in the legs which can have considerable bearing on the yield and fatigue strength of the jack-up. Hence, suitable modifications to the present classification rules and guidelines are required to account for the effect of VIV in the design of jack-ups. The mathematical approach presented will enable practicing engineers to effectively consider conservatively the effect of VIV due to regular waves in jack-up designs. The test results also emphasise the significance of yaw or torsional VIV in regular waves.

\section{ACKNOWLEDGEMENTS}

This work was carried out by using the facilities at the School of Marine Sciences and Technology, Newcastle University, UK and was supported by M/s Cybermarine Technologies Pte Ltd, Singapore. The authors gratefully acknowledge the unconditional support provided by both Newcastle University and Cybermarine Technologies.

\section{REFERENCES}

[1] Sarpkaya, T., and Isaacson, M., 1981, Mechanics of wave forces on offshore structures, Van Nostrand Reinhold Co.

[2] Blevins, R. D., 2001, Flow-Induced Vibration, Krieger Publishing Company, Florida.

[3] Sarpkaya, T., 2004, "A critical review of the intrinsic nature of vortex-induced vibrations," Journal of Fluids and Structures, 19(4), pp. 389-447.

[4] Williamson, C. H. K., and Govardhan, R., 2004, "VORTEXINDUCED VIBRATIONS," Annual Review of Fluid Mechanics, 36(1), pp. 413-455.

[5] Sumer, B. M., and Fredsøe, J., 2006, Hydrodynamics around Cylindrical Structures (Revised Edition), World Scientific.

[6] Khalak, A., and Williamson, C. H. K., 1997, "Investigation of relative effects of mass and damping in vortex-induced vibration of a circular cylinder," Journal of Wind Engineering and Industrial Aerodynamics, 69-71(0), pp. 341-350.

[7] Govardhan, R., and Williamson, C. H. K., 2004, "Critical mass in vortex-induced vibration of a cylinder," European Journal of Mechanics - B/Fluids, 23(1), pp. 17-27. 
[8] Vandiver, J. K., 2012, "Damping Parameters for flowinduced vibration," Journal of Fluids and Structures, 35, pp. 105119.

[9] Gonçalves, R. T., Rosetti, G. F., Fujarra, A. L. C., Nishimoto, K., and Oliveira, A. C., 2011, "Experimental Study on VortexInduced Motions (VIM) of a Large-Volume Semi-Submersible Platform," (44397), pp. 1-9.

[10] Gonçalves, R. T., Fujarra, A. L. C., Rosetti, G. F., Kogishi, A. M., and Koop, A., 2018, "Experimental study of the column shape and the roughness effects on the vortex-induced motions of deep-draft semi-submersible platforms," Ocean Engineering, 149, pp. 127-141.

[11] Zdravkovich, M. M., 1985, "Flow induced oscillations of two interfering circular cylinders," J Sound Vib, 101(4), pp. 511521.

[12] Wang, X. K., Gong, K., Liu, H., Zhang, J. X., and Tan, S. K., 2013, "Flow around four cylinders arranged in a square configuration," Journal of Fluids and Structures, 43(0), pp. 179199.

[13] Assi, G. R. S., Bearman, P. W., and Meneghini, J. R., 2010, "On the wake-induced vibration of tandem circular cylinders: the vortex interaction excitation mechanism," J. Fluid Mech., 661, pp. 365-401.

[14] Jiang, R., 2012, "FLOW-INDUCED VIBRATIONS OF TWO TANDEM CYLINDERS IN A CHANNEL," Thermal Science, 16(5), pp. 1377-1381.

[15] Zhao, M., and Cheng, L., 2012, "Numerical simulation of vortex-induced vibration of four circular cylinders in a square configuration," Journal of Fluids and Structures, 31(0), pp. 125140.

[16] Angrilli, F., and Cossalter, V., 1982, "Transverse Oscillations of a Vertical Pile in Waves," Journal of Fluids Engineering, 104(1), pp. 46-52.

[17] Bearman, P. W., Graham, J. M. R., and Obasaju, E. D., 1984, "A model equation for the transverse forces on cylinders in oscillatory flows," Applied Ocean Research, 6(3), pp. 166-172.

[18] Sumer, B. M., and Fredsok, J., 1988, "Transverse Vibrations of an Elastically Mounted Cylinder Exposed to an Oscillating Flow," Journal of Offshore Mechanics and Arctic Engineering, 110(4), pp. 387-394.

[19] McConnell, K. G., and Park, Y. S., 1982, "The frequency components of fluid-lift forces acting on a cylinder oscillating in still water," Experimental Mechanics, 22(6), pp. 216-222.

[20] Sarpkaya, T., 1975, "Forces on Cylinders and Spheres in a Sinusoidally Oscillating Fluid," Journal of Applied Mechanics, 42(1), pp. 32-37.

[21] Verley, R. L. P., 1982, "A simple model of vortex-induced forces in waves and oscillating currents," Applied Ocean Research, 4(2), pp. 117-120.

[22] Sarpkaya, T., 1987, "Oscillating Flow About Smooth and Rough Cylinders," Journal of Offshore Mechanics and Arctic Engineering, 109(4), pp. 307-313.

[23] Maull, D. J., and Milliner, M. G., 1978, "Sinusoidal flow past a circular cylinder," Coastal Engineering, 2, pp. 149-168.

[24] Hayashi, K., Higaki, F., Shigemura, T., and Chaplin, Jr., 2003, "Vortex-excited vibration of a circular cylinder in planar oscillating flow," International Journal Of Offshore And Polar Engineering, 13(4), pp. 266-273.

[25] Chaplin, J. R., 1988, "Loading on a cylinder in uniform oscillatory flow: Part I- Planar oscillatory flow," Applied Ocean Research, 10(3), pp. 120-128.

[26] Sumer, B. M., Fredøe, J., and Jensen, K., 1994, "A Note on Spanwise Correlation on a Freely Vibrating Cylinder in Oscillatory Flow," Journal of Fluids and Structures, 8(3), pp. 231-238.

[27] Blevins, R. D., and Burton, T. E., 1976, "Fluid Forces Induced by Vortex Shedding," Journal of Fluids Engineering, 98(1), pp. 19-24.

[28] Iwan, W. D., and Blevins, R. D., 1974, "A Model for Vortex Induced Oscillation of Structures," Journal of Applied Mechanics, 41(3), pp. 581-586.

[29] Skop, R. A., and Griffin, O. M., 1973, "A model for the vortex-excited resonant response of bluff cylinders," J Sound Vib, 27(2), pp. 225-233.

[30] Barltrop, N. D. P., and Adams, A. J., 1991, Dynamics of Fixed Marine Structures (Third Edition), ButterworthHeinemann.

[31] Vickery, B. J., and Watkins, R. D., 1964, "FLOWINDUCED VIBRATIONS OF CYLINDRICAL STRUCTURES A2 - SILVESTER, RICHARD," Hydraulics and Fluid Mechanics, Pergamon, pp. 213-241.

[32] Nicholls-Lee, R., Hindley, S., and Parkinson, R., "Development of an economic and efficient installation vessel for tidal stream energy converter arrays," Proc. International Conference on Offshore Mechanics and Arctic Engineering OMAE, ASME, p. 9.

[33] Thake, J., 2005, "Development, Installation and Testing of a Large-Scale Tidal Current Turbine ", Department of Trade and Industry, UK.

[34] Bennett Jr, W. T., and Patel, R. K., 1989, "Jack-up behavior in elevated condition: model test and computer simulation," SNAME, NJ.

[35] Johnson, T. L., and Patel, R. K., 1992, "The use of small scale physical models and numerical models for jack-up design," Recent Developments in Jack-up Platforms, Blackwell Scienific Publications, Oxford.

[36] Grundlehner, G. J., 1997, "Systematic model tests on a harsh environment jack-up in elevated condition," Marine Structures, 10(2-4), pp. 159-180.

[37] Ramadasan, S., Tao, L., and Dev, A., 2018, "Antinode Fairings: An Optimum Solution for Reduction of Vortex Induced Vibration," Offshore Technology Conference Asia, Offshore Technology Conference, Kuala Lumpur, Malaysia.

[38] DNV.GL, 2017, DNVGL-RP-C205, Recommended Practice, Environmental Conditions and Environmental Loads, DNV GL AS, Oslo.

[39] Williamson, C. H. K., 1985, "Sinusoidal flow relative to circular cylinders," Journal of Fluid Mechanics, 155, pp. 141174. 